\title{
Electronic Excited State Lifetimes of Anionic Water Clusters: Dependence on Charge Solvation Motif
}

\author{
Daniel Borgis, ${ }^{\dagger}$ Peter J. Rossky, ${ }^{\ddagger}$ and László Turi* ${ }^{\star}{ }^{(0)}$ \\ ${ }^{\dagger}$ Pôle de Chimie Théorique, UMR-CNRS PASTEUR, Ecole Normale Supérieure, 24, rue Lhomond, 75231 Paris Cedex 05, France \\ ${ }^{\ddagger}$ Department of Chemistry, Rice University, P.O. Box 1892, MS-60, Houston, Texas 77251-1892, United States \\ ${ }^{\S}$ Department of Physical Chemistry, ELTE Eötvös Loránd University, Budapest 112, P.O. Box 32, H-1518 Budapest, Hungary
}

\section{Supporting Information}

ABSTRACT: An ongoing controversy about water cluster anions concerns the electronbinding motif, whether the charge center is localized at the surface or within the cluster interior. Here, mixed quantum-classical dynamics simulations have been carried out for a wide range of cluster sizes $(n \leq 1000)$ for $\left(\mathrm{H}_{2} \mathrm{O}\right)_{n}^{-}$and $\left(\mathrm{D}_{2} \mathrm{O}\right)_{n}^{-}$, based on a nonequilibrium first-order rate constant approach. The computed data are in good general agreement with time-resolved photoelectron imaging results $(n \leq 200)$. The analysis reveals that, for surface state electrons, the cluster size dependence of the excited state electronic energy gap and the magnitude of the nonadiabatic couplings have compensating influences on the excited state lifetimes: the excited state lifetime for surface states reaches a minimum for $n \sim 150$ and then increases for larger clusters. It is concluded that the electron resides in a surface-localized motif in all of these measured clusters, dominating at least up to $n=200$.

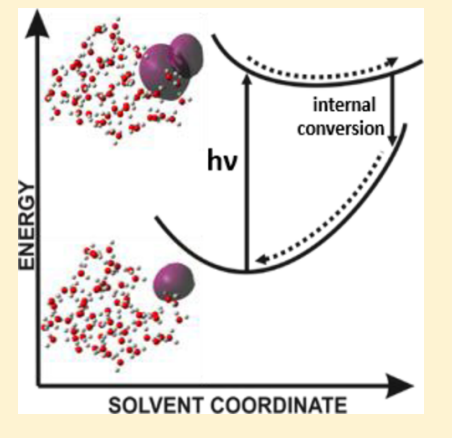

$\mathrm{E}$ xcess electrons in aqueous environments are known as hydrated electrons. ${ }^{1}$ Excess electron hydration in bulk water and in finite size water clusters have important implications as models for the electronic response to solvation in various physical, chemical, and biological areas. ${ }^{2-4}$ For this reason, hydrated electrons and water cluster anions have been popular targets for the most advanced ultrafast spectroscopic $^{5-11}$ and theoretical investigations. ${ }^{12-19}$

Water cluster anions, in particular, have been the focus of intensive scientific scrutiny in the past decade. ${ }^{6-8,16-18,20,21}$ It has been established that finite size hydrated electron systems form several experimentally distinguishable homologous sequences with cluster size, depending on the conditions of cluster formation. $^{8,22,23}$ The so-called isomer I, which was that observed in the earliest experiments, ${ }^{24}$ binds the electron most strongly for any given cluster size, and has been the subject of the most detailed experimental studies of dynamics. ${ }^{8,20,25}$ In parallel, two main solvation motifs for stable clusters were established in thermal cluster simulations, predicting the extra electron to bind either localized at the surface (surface state clusters) or in the interior (interior state clusters), the latter being analogous to the hydrated electron in bulk water. ${ }^{26}$ The different structural motifs may, in principle, lead to detectable differences in the measurable physical quantities. ${ }^{16,27}$ Nevertheless, the identification of the common structural motifs within the groups of isomers is still a subject of considerable debate. ${ }^{8,16-18,22,27-29}$ The essential difficulty lies in the fact that the experimental conditions of cluster preparation profoundly influence the outcome, as has been demonstrated both experimentally ${ }^{8}$ and theoretically. ${ }^{30}$ Hence, the possibility that some set of these isomers represents nonequilibrium trapped states is a substantial complication. Since the isomer I class is apparently the only one formed at relatively warmer cluster conditions, ${ }^{8,22,25}$ a thermal equilibrium theory of cluster properties can plausibly be best compared with experimental isomer I results. It is this cluster type that will be our focus here.

In addition to energetic and static spectroscopic probes, dynamic signatures offer an additional window that might distinguish cluster anion structures. One such feature, which has been studied in considerable detail in the bulk,,$^{5,31-35}$ is the electronic excited state lifetime following photoexcitation. For water cluster anions, this might be expected to vary with cluster size. The earliest such measurement could only report that the excited state lifetime of moderate size clusters was comparable to the laser pulse width of $150 \mathrm{fs} .{ }^{36}$ Paik et al. ${ }^{6}$ observed two time scales ( $300 \mathrm{fs}$ and $2-10 \mathrm{ps}$ ) in time-resolved photoelectron spectroscopy (PES) experiments on water cluster anions $\left(\mathrm{H}_{2} \mathrm{O}\right)_{n}^{-}$with $n=15-35$, and attributed these times to ground state solvent relaxation following nonadiabatic transition, as suggested in one scenario for the bulk. ${ }^{5}$ The Neumark group has performed a comprehensive series of time-resolved photoelectron imaging (TR-PEI) experiments on $\left(\mathrm{H}_{2} \mathrm{O}\right)_{n}^{-}$and $\left(\mathrm{D}_{2} \mathrm{O}\right)_{n}^{-}$clusters for a wide range, $n=25-200 .{ }^{8,22}$ The excited state lifetimes convincingly extracted from the data over the whole size range for isomer I clusters are distinctly sizedependent (decreasing with increasing cluster size) and show a quite significant solvent isotope effect. Extrapolation of type I clusters to the infinite size limit results in $\sim 60$ fs excited state lifetime. $\mathrm{D}_{2} \mathrm{O}$ water clusters relax more slowly than $\mathrm{H}_{2} \mathrm{O}$ by a factor of $\sim 2-3$, increasing with cluster size. ${ }^{8,22}$ More recently,

Received: March 7, 2017

Accepted: May 5, 2017

Published: May 5, 2017 
time-resolved photoemission measurements have been reported by two groups for electronic excited state internal conversion rates using hydrated electrons created in water microjets. ${ }^{37,38}$ Those groups reported similar lifetime estimates, $\sim 60 \mathrm{fs}^{38}$ and $\sim 75 \mathrm{fs}^{37}$ in $\mathrm{H}_{2} \mathrm{O}$, comparable to that reported in the largest measured clusters ${ }^{22}$ and inferred in the bulk from photon echo measurements earlier. ${ }^{34} \mathrm{We}$ will consider reconciliation of the present results with these recent reports based on microjets in the ensuing discussion.

Up to now, theory has not rationalized the observed trends for the excited state lifetimes of water cluster anions nor completed the connection of these observations with bulk hydrated electron excited state dynamics. In this report, we present a molecular level description of the electronic dynamics and solvent relaxation for thermal equilibrium anionic water clusters that makes it possible to associate the experimentally observed isomer I lifetimes with theoretically predicted structural characteristics, and to develop generalizable insights into radiationless transition phenomena in cluster and solution environments.

The calculation of the lifetime of the excited state water cluster anions is based on the quantized autocorrelation function approach developed by us and applied successfully for estimating the excited state lifetime of the bulk hydrated electron. ${ }^{39,40}$ Here, the quantum mechanical expression for the first-order perturbation theory rate of radiationless transition, Fermi's Golden Rule, for the nonadiabatic transition between two electronic states $1 \rightarrow 2$ is utilized in its time-dependent form. The fundamental quantities needed for a given system are the quantum time autocorrelation functions of (a) the $E_{1}-E_{2}$ energy gap and (b) the quantum mechanical nonadiabatic coupling between the states (see Supporting Information, eqs $\mathrm{S} 1-\mathrm{S} 3$ ). The central quantities that need to be evaluated from trajectory simulations are thus the history of the energy gaps, $\hbar \Omega_{12}(t)$, following an initial, essentially instantaneous, transition to the lowest electronic excited state, the corresponding temporal history of the nonadiabatic (NA) coupling matrix elements, $V_{12}(t)$, and the normalized autocorrelation functions, $\tilde{C}_{\Omega}(t)$ and $\tilde{C}_{V}(t)$, and their Fourier transforms, $\tilde{C}_{\Omega}(\omega)$ and $\tilde{C}_{V}(\omega)$, which follow from these histories. The usual Golden Rule formalism prescribes that this should be done for an equilibrium ensemble of the system in the initial electronic state 1 , with a monitored mean energy gap $\left\langle\Omega_{12}\right\rangle{ }^{39}$ In the experimental photoexcitation experiment, the excess electron is promoted to the excited state and finds itself in a nonequilibrium solvent environment, initially still equilibrated with the now unoccupied ground state electron distribution. The solvent relaxation that follows gradually decreases the energy gap, and the decreasing gap accelerates the electronic transition rate, as seen in simulations of the bulk $\mathrm{e}_{\mathrm{aq}}{ }^{-39-41}$ Thus, to compute the lifetime of the electron in the excited state, the coupled solvent relaxation and electronic transition must be taken into account. ${ }^{40}$ These computed lifetimes can be directly compared to the lifetimes observed in the $\sim 100 \mathrm{fs}$ time resolution experiments of Neumark et al. ${ }^{8,22}$ The details of the simulations and the evaluation of the data are given in the Supporting Information.

First, we illustrate the general behavior of relevant characteristics for the surface and interior state isomers of the $n=500$ water cluster anion. As anticipated, the distributions of the energy gap (top panel, Figure 1) closely follow a Gaussian form, similar to the bulk hydrated electron case. ${ }^{40}$ It is important to notice that the characteristic energy gaps are

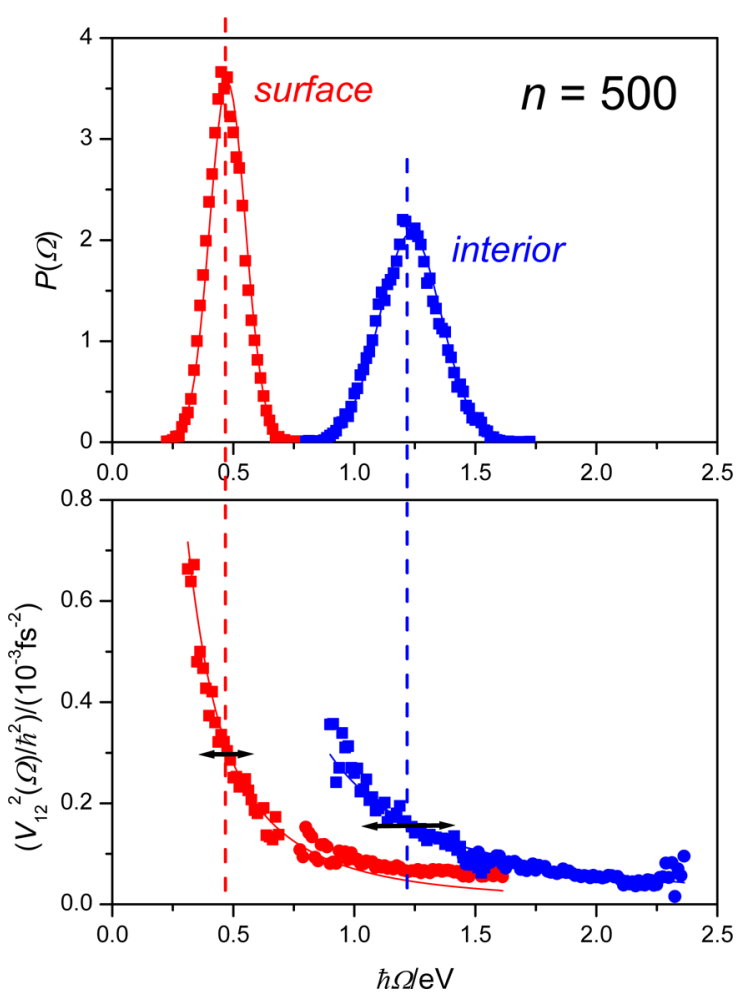

Figure 1. Ground to first electronic excited state energy gap distribution (top panel) and energy gap dependence of the squared nonadiabatic coupling (bottom panel) for surface states (red) and interior states (blue) for simulated $\left(\mathrm{H}_{2} \mathrm{O}\right)_{500}^{-}$clusters. The energy gap distribution is taken from a representative portion of midstate simulations; the squared NA coupling distributions are from ground state (squares) and midstate simulations (circles). The continuous curves show the Gaussian approximations (top), and the $V_{12}^{2}(\Omega)=a$ / $(\hbar \Omega)^{2}$ fit (bottom, $a=7.0 \times 10^{-5}$ for surface and $2.4 \times 10^{-4}$ for interior states). The vertical dashed lines guide the eye to the nonadiabatic coupling in the bottom panel at the most probable energy gap marked by horizontal arrows; the gap full width at halfmaximum is indicated by the length of the arrows.

significantly smaller for surface states (summarized in Table S1 in the Supporting Information), as has been observed through the spectra of water cluster anions. ${ }^{16,27}$ The squares of the gapdependent coupling $\left\langle V_{12}^{2}(\Omega)\right\rangle$ (bottom panel, Figure 1) do lie on a single curve, whether from ground state or midstate surface simulations, and can be fitted with a simple oneparameter equation for each cluster size separately. ${ }^{40}$ Figure 1 reveals critical observations that will underlie the interpretation of the lifetime data. In particular, it is apparent that the interior state clusters have larger NA couplings at any given energy gap, due to the larger number of molecules in the vicinity of the electron, as one should anticipate, while the surface state clusters exhibit anticipated smaller energy gaps. However, when considering the two aspects in combination, Figure 1 clearly shows the critical fact that the NA couplings are generally greater for surface states than interior states at the average energy gaps that are predominantly populated.

The coupling correlation functions are highly oscillatory due to the role of molecular vibrational momenta and are more readily discussed in the frequency domain. The spectral density of these functions (not shown), similar to the bulk hydrated electron case, indicates strong coupling to librational and vibrational modes of the aqueous environment. ${ }^{40}$ The energy gap correlation functions (Figures S1 and S2 of the Supporting 
Information) are rather uniform in their dynamics (within statistical uncertainty), irrespective of the cluster size and the electron localization mode, suggesting that the solvent dynamics in the vicinity of the electron is largely similar in each environment. For this reason, we constructed a single master curve for the normalized energy gap autocorrelation function for each solvent (i.e., $\mathrm{H}_{2} \mathrm{O}, \mathrm{D}_{2} \mathrm{O}$ ) from the average of the individual cluster gap correlation functions obtained from simulation. The individual autocorrelation functions and the master curves for water and heavy water are collected in Figures S1 and S2. The master curve is described analytically by a typical solvation dynamics form (Gaussian plus biexponential function), which is then used in the lifetime calculations. The Gaussian inertial regime reflects a solvent isotope effect, ${ }^{5}$ with time constants of 10 and 14 fs for $\mathrm{H}_{2} \mathrm{O}$ and $\mathrm{D}_{2} \mathrm{O}$, respectively. To quantify uncertainties in the gap correlation functions, we calculated the correlation functions that represent the $95 \%$ confidence interval of the master curves. We then describe this family of curves with respect to the (mean) master curve using a parametrized model (see Supporting Information). There we show that variations corresponding to statistical errors do not affect the conclusions of this study, and the corresponding uncertainties are reported with the data, below.

Using the above quantities, it is possible to evaluate the gapdependent rate expression (eq S7). Figure 2 illustrates the computed lifetime $\left(\tau_{1 \rightarrow 2}(\bar{\Omega}(t))=1 / k_{1 \rightarrow 2}(\bar{\Omega}(t))\right)$ function for selected water cluster anions (top panel) and the mean energy gap as a function of inverse cluster size (bottom panel). As anticipated, small energy gaps induce faster transition for a given cluster size and type, and larger clusters have shorter
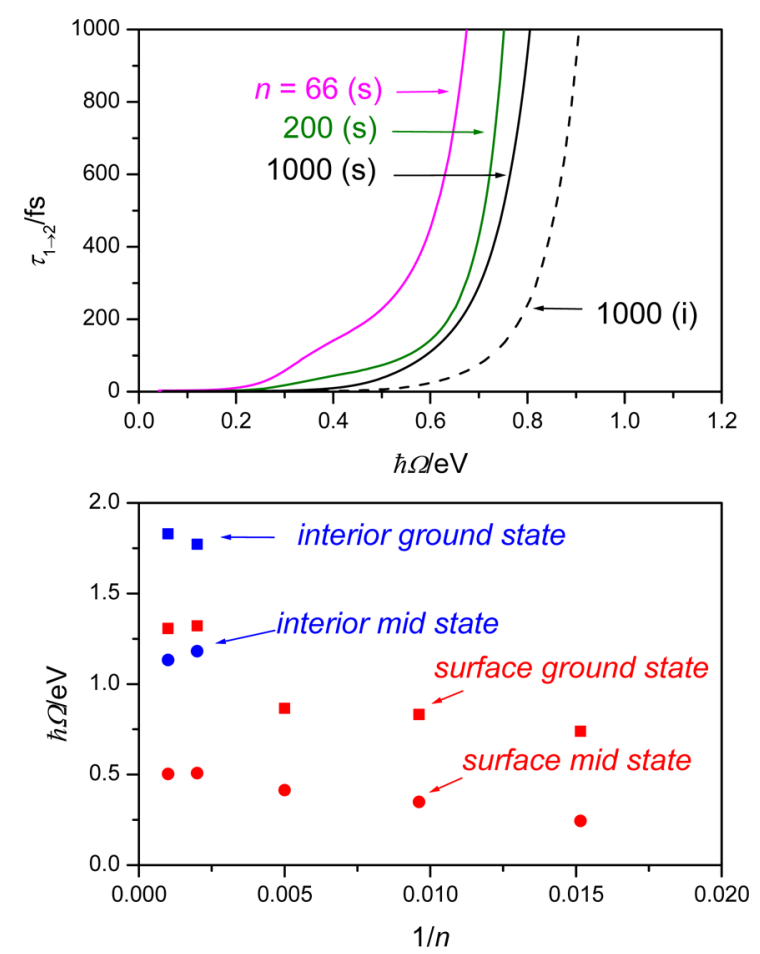

Figure 2. Equilibrium excited state lifetimes, $\tau_{1 \rightarrow 2}\left(\left\langle\Omega_{12}\right\rangle\right)=1 /$ $k_{1 \rightarrow 2}\left(\left\langle\Omega_{12}\right\rangle\right)$, for different sized $(n=66,200,1000) \mathrm{H}_{2} \mathrm{O}$ clusters, calculated as a function of the mean energy gap. Solid curves: surface (s) states; dashed curves: interior (i) states. The bottom panel shows the mean energy gap as a function of inverse cluster size from ground state and from midstate simulations. excited state lifetimes for a given energy gap. We may also notice that interior states are faster to relax at the same gap than surface state clusters, due to their larger NA coupling contribution (see Figure 1). Thus, the excited state lifetime for equilibrium interior states at the estimated $\sim 0.4 \mathrm{eV}$ equilibrium gap of the excited state bulk hydrated electron would be predicted to be roughly $10 \mathrm{fs}$. The excited state lifetime of the equilibrium surface state clusters at the same gap size would be much larger, although still under $100 \mathrm{fs.}$

Let us now consider the calculation of the survival probability function of eq $\mathrm{S} 7$ for the excited state lifetime of clusters. The calculated survival probability functions for $n=500$ surface and interior state clusters (Figure 3) demonstrate that the relative

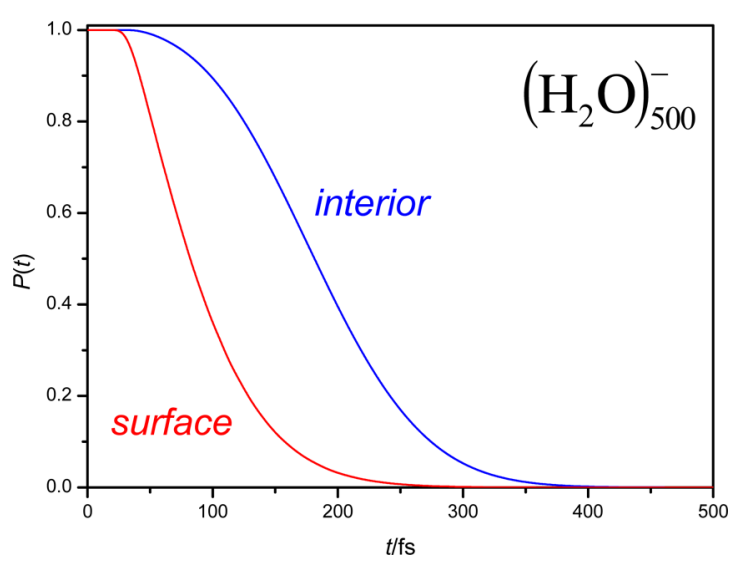

Figure 3. Electronic excited state survival probability as a function of time for $\left(\mathrm{H}_{2} \mathrm{O}\right)_{500}^{-}$clusters for surface (red) and interior (blue) states.

size of excited state lifetimes reverses if one takes the energy gap dynamics and the corresponding transition rate dependence into account. Assuming no important differences in solvent relaxation rate, as inferred from the energy gap correlation functions, excited state surface clusters relax electronically much faster than their interior state counterparts, due to their significantly smaller energy gaps. This factor clearly dominates the lifetime. Computed excited state lifetimes in $\left(\mathrm{H}_{2} \mathrm{O}\right)_{500}^{-}$clusters are $\sim 200 \mathrm{fs}$ for the surface state and $\sim 500 \mathrm{fs}$ for the interior state cluster, the latter comparable to the value computed previously for bulk $\mathrm{e}_{\mathrm{aq}}{ }^{-40}$, with the former noticeably shorter.

The lifetimes for all the clusters simulated here are collected in Figure 4, along with experimental data from ref 22. The computed lifetime for surface state clusters falls in the $\sim 70-$ $200 \mathrm{fs}$ region. Within the surface state group, the tendency for faster transition due to their larger couplings in larger clusters is compensated by the smaller gap in smaller clusters, so that the size dependence is both quite weak and nonmonotonic over the range accessible to theory. We note that the appreciably increased gaps for the $n=500$ and 1000 surface state clusters compared to smaller clusters also indicate a somewhat deeper penetration of the excess electronic wave function in the interior of these clusters. The two large interior state clusters investigated have a significantly longer lifetime at $\sim 400-500 \mathrm{fs}$, consistent with the simulated lifetime estimate for a bulk hydrated electron, $330 \mathrm{fs}^{40}$ (using the same theory at a higher temperature of $300 \mathrm{~K}$ ), and with the values inferred from pump-probe experiments ${ }^{5}$ and independent excited state electron scavenging kinetics, ${ }^{10,42}$ which put the electronic 

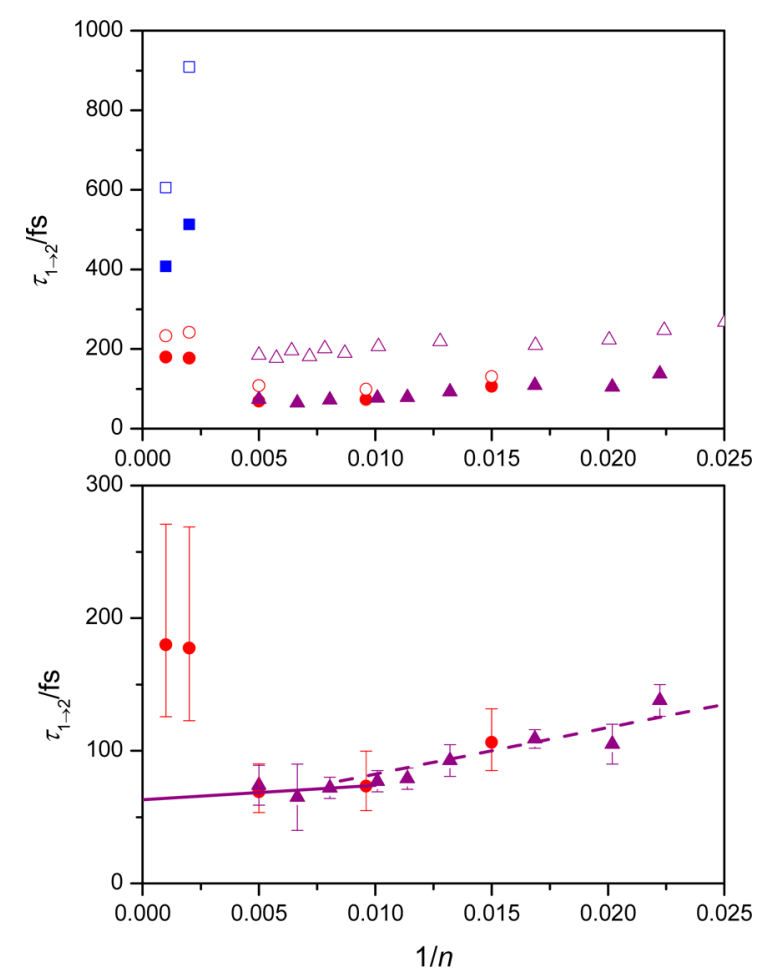

Figure 4. Calculated electronic excited state lifetime as a function of inverse cluster size $(1 / n)$ for $\left(\mathrm{H}_{2} \mathrm{O}\right)_{n}^{-}$(filled symbols) and $\left(\mathrm{D}_{2} \mathrm{O}\right)_{n}^{-}$ (open symbols). Simulated surface states are indicated by red, interior states by blue. Experimental results (purple) from ref 22 are shown for type $\mathrm{I}$ isomer clusters for $\mathrm{H}_{2} \mathrm{O}$ (filled triangles) and for $\mathrm{D}_{2} \mathrm{O}$ (open triangles). The bottom panel shows the $\mathrm{H}_{2} \mathrm{O}$ results on an expanded lifetime axis $(\tau \leq 300 \mathrm{fs})$, and includes both reported experimental ${ }^{22}$ and simulation error bars, as well as the reported linear fits of experimental data ${ }^{22}$ for $n \leq 70$ (dashed line) and $n>70$ (solid line).

excited state lifetime of the bulk excited state hydrated electron in the $\sim 300-500 \mathrm{fs}$ range.

Deuteration of the solvent does increase the computed lifetimes in all cases, but the trends for surface versus interior state lifetimes are the same as in light water. From a quantitative perspective, the comparison to experiment ${ }^{8,22}$ yields agreement between theory and experiment for $\mathrm{H}_{2} \mathrm{O}$ clusters that is extremely good, agreement that must be assumed to be, in part, fortuitous (Figure 4, bottom panel). The calculated isotope effect (factor of 1.3-1.5) is underestimated, compared to the experimental reports, for the clusters for which comparison can be made, although it is of the same magnitude (Figure 4, top panel). At the same time, we note that an application of the same theory used here to the bulk hydrated electron yields an isotope effect of a factor of $\sim 2$ on the lifetime, while experiment ${ }^{32}$ does not reveal a significant isotope effect. We infer that the most likely origin of the quantitative differences lies in the approximate harmonic nuclear quantization scheme incorporated in the current theory. ${ }^{39,40}$ Nevertheless, one cannot rule out a contribution due to differences in the properties between the experimental $\mathrm{H}_{2} \mathrm{O}$ versus $\mathrm{D}_{2} \mathrm{O}$ clusters, such as a difference in effective cluster temperature. ${ }^{25}$

Considering the direct comparison with experiment ${ }^{8,22}$ displayed in Figure 4, it appears that the extrapolation of cluster lifetimes to the bulk case suggested in ref 22 (reproduced in Figure 4, bottom panel) is a consequence of the slowly varying lifetimes of clusters near their minimum in the vicinity of $n \sim 150$. The data also show that the experimental trend for isomer I is closely reproduced by the simulated surface state clusters, and is not consistent with the simulated larger $(n \geq 500)$ interior state clusters; the latter, with consistently larger energy gaps, ${ }^{16,27}$ invariably have notably longer lifetimes for the same cluster size and nominal temperature. This observation reinforces earlier studies of the size dependence of the absorption spectrum and radius of the excess electron ${ }^{16,27}$ that indicated that the experimentally observed type I clusters are consistent with a binding motif with the excess electron resident at the surface of the clusters and inconsistent with an interior state. The present study extends this conclusion up to $n=200$ and provides an additional predicted experimental signature, along with distinct absorption spectral shifts, ${ }^{16}$ for the appearance of an interior bound electron, namely, a large increase in excited state lifetime. Finally, the extrapolation of the experimental data to infinite size "bulk" clusters, yielding an ultrashort lifetime for $\mathrm{e}_{\mathrm{aq}}{ }^{-}$in the bulk of $\sim 60 \mathrm{fs},{ }^{22}$ has been argued to be consistent with a reported experimental inference of a comparable excited state lifetime, ${ }^{34,38,43}$ and thus to support the so-called "non-adiabatic model" for relaxation. ${ }^{20}$ In that model, the brief dynamics on the excited state surface has little effect on the observed spectral dynamics; the spectral evolution is dominated by hot state relaxation on the ground state surface following the nonadiabatic transition. The conclusions we have presented in the current work do not support that line of reasoning for inferring mechanistic aspects for the bulk $\mathrm{e}_{\mathrm{aq}}{ }^{-}$. Nevertheless, the nonadiabatic model may well describe the electronic dynamics for surface state clusters with ultrashort lifetimes.

Given the recent assignment of sub-100 fs excited state lifetimes for hydrated electrons in water jets, ${ }^{37,38}$ one might be inclined to dismiss the current calculation as that of a model in fortuitous agreement with a measurement. We suggest that there are also reasons to be uncertain about the experimental assignment, considering the experimental bulk solution measurements that have convincingly used chemistry along with lifetime measurements to assign a much longer $\mathrm{e}_{\mathrm{aq}}{ }^{-*}$ lifetime. $^{10,42}$ The electrons are observed in the microjet only 200 ps after their photoexcitation of iodide ${ }^{37}$ or bromide, ${ }^{38}$ both large halides whose interfacial propensity is readily observed. ${ }^{44}$ Hence, the formation of near surface species should be a significant population. Further, it has been clearly noted by Karashima et al. ${ }^{38}$ that elastic scattering with solvent should yield isotropic photoemission, but photoemission from the observed excited state is initially anisotropic. The observation has been attributed ${ }^{38}$ to a "larger electron density for the ES [excited state] at the top molecular layer of the liquid." Finally, there is increasing evidence also based in part on observed photoelectron anisotropy, that the electron probing depth of near threshold photoemission is much shorter than previously assumed. ${ }^{45}$ Recent measured photoemission data from aerosol water drops ${ }^{46}$ can be modeled consistently with a low energy photoelectron mean free path of $\sim 5 \mathrm{~nm}$; in principle, then, signals would include species well below the interface. At the same time, theoretical TDDFT calculations ${ }^{47}$ indicate that the equilibrated interfacial solvated electron has an excitation gap comparable to the bulk; this "infinite" cluster surface state result is consistent with the trend we see with increasing size (see Figure 4), suggesting, based on scavenging experiments, ${ }^{10,42}$ that a quasi-equilibrated interfaceresident hydrated electron should not have an ultrafast lifetime. Given that the cluster observations in the present work are explained by physically evident and transparently sensible 
opposing trends in energy gaps and nonadiabatic couplings and agree with experiment, while uncertainties exist as to the species observed in microjet experiments, we believe that there are good reasons to weigh both these calculations and recent experiments in this ongoing and still unresolved debate.

\section{ASSOCIATED CONTENT}

\section{S Supporting Information}

The Supporting Information is available free of charge on the ACS Publications website at DOI: 10.1021/acs.jpclett.7b00555.

Details of the molecular dynamics simulations, the theoretical background for the data evaluation, and the calculation of error estimates (PDF)

\section{AUTHOR INFORMATION}

\section{Corresponding Author}

*E-mail: turi@chem.elte.hu.

\section{ORCID ${ }^{\circ}$}

Peter J. Rossky: 0000-0002-0461-4179

László Turi: 0000-0001-8238-7816

Notes

The authors declare no competing financial interest.

\section{ACKNOWLEDGMENTS}

P.J.R. acknowledges the support of this research by the U.S. National Science Foundation (CHE-0910499, CHE-1362381). This work was supported by a research grant to LT from the National Research, Development and Innovation Office, Hungary (NKFIH, previously OTKA, K104237).

\section{REFERENCES}

(1) Hart, E. J.; Boag, J. W. Absorption Spectrum of the Hydrated Electron in Water and in Aqueous Solutions. J. Am. Chem. Soc. 1962, 84, 4090-4095.

(2) Hart, E. J.; Anbar, M. The Hydrated Electron; John Wiley \& Sons: New York, 1970.

(3) Garrett, B. C.; Dixon, D. A.; Camaioni, D. M.; Chipman, D. M.; Johnson, M. A.; Jonah, C. D.; Kimmel, G. A.; Miller, J. H.; Rescigno, T. N.; Rossky, P. J.; et al. Role of Water in Electron-Initiated Processes and Radical Chemistry: Issues and Scientific Advances. Chem. Rev. (Washington, DC, U. S.) 2005, 105, 355-390.

(4) Siefermann, K. R; Abel, B. The Hydrated Electron: A Seemingly Familiar Chemical and Biological Transient. Angew. Chem., Int. Ed. 2011, 50, 5264-5272.

(5) Silva, C.; Walhout, P. K.; Yokoyama, K.; Barbara, P. F. Femtosecond Solvation Dynamics of the Hydrated Electron. Phys. Rev. Lett. 1998, 80, 1086-1089.

(6) Paik, D. H.; Lee, I.-R.; Yang, D.-S.; Baskin, J. S.; Zewail, A. H. Electrons in Finite-Sized Water Cavities: Hydration Dynamics Observed in Real Time. Science 2004, 306, 672-675.

(7) Hammer, N. I.; Shin, J.-W.; Headrick, J. M.; Diken, E. G.; Roscioli, J. R.; Weddle, G. H.; Johnson, M. A. How Do Small Water Clusters Bind an Excess Electron? Science 2004, 306, 675-679.

(8) Verlet, J. R. R.; Bragg, A. E.; Kammrath, A.; Cheshnovsky, O.; Neumark, D. M. Observation of Large Water-Cluster Anions with Surface-Bound Excess Electrons. Science 2005, 307, 93-96.

(9) Li, B.; Zhao, J.; Onda, K.; Jordan, K. D.; Yang, J.; Petek, H. Ultrafast Interfacial Proton-Coupled Electron Transfer. Science 2006, 311, 1436-1440.

(10) Wang, C.-R.; Lu, Q.-B. Real-Time Observation of a Molecular Reaction Mechanism of Aqueous 5-Halo-2'-deoxyuridines under UV/ Ionizing Radiation. Angew. Chem., Int. Ed. 2007, 46, 6316-6320.

(11) Siefermann, K. R.; Liu, Y.; Lugovoy, E.; Link, O.; Faubel, M.; Buck, U.; Winter, B.; Abel, B. Binding Energies, Lifetimes and
Implications of Bulk and Interface Solvated Electrons in Water. Nat. Chem. 2010, 2, 274-279.

(12) Barnett, R. N.; Landman, U.; Cleveland, C. L.; Jortner, J. Surface States of Excess Electrons on Water Clusters. Phys. Rev. Lett. 1987, 59, 811-814.

(13) Schnitker, J.; Motakabbir, K.; Rossky, P. J.; Friesner, R. A Priori Calculation of the Optical-Absorption Spectrum of the Hydrated Electron. Phys. Rev. Lett. 1988, 60, 456-459.

(14) Boero, M.; Parrinello, M.; Terakura, K.; Ikeshoji, T.; Liew, C. C. First-Principles Molecular-Dynamics Simulations of a Hydrated Electron in Normal and Supercritical Water. Phys. Rev. Lett. 2003, 90, 226403.

(15) Jordan, K. D.; Wang, F. Theory of Dipole-Bound Anions. Annu. Rev. Phys. Chem. 2003, 54, 367-396.

(16) Turi, L.; Sheu, W.-S.; Rossky, P. J. Characterization of Excess Electrons in Water-Cluster Anions by Quantum Simulations. Science 2005, 309, 914-917.

(17) Jacobson, L. D.; Herbert, J. M. Theoretical Characterization of Four Distinct Isomer Types in Hydrated-Electron Clusters, and Proposed Assignments for Photoelectron Spectra of Water Cluster Anions. J. Am. Chem. Soc. 2011, 133, 19889-19899.

(18) Barnett, R. N.; Giniger, R.; Cheshnovsky, O.; Landman, U. Dielectron Attachment and Hydrogen Evolution Reaction in Water Clusters. J. Phys. Chem. A 2011, 115, 7378-7391.

(19) Marsalek, O.; Uhlig, F.; VandeVondele, J.; Jungwirth, P. Structure, Dynamics, and Reactivity of Hydrated Electrons by $\mathrm{Ab}$ Initio Molecular Dynamics. Acc. Chem. Res. 2012, 45, 23-32.

(20) Young, R. M.; Neumark, D. M. Dynamics of Solvated Electrons in Clusters. Chem. Rev. (Washington, DC, U. S.) 2012, 112, 55535577.

(21) Turi, L.; Rossky, P. J. Theoretical Studies of Spectroscopy and Dynamics of Hydrated Electrons. Chem. Rev. (Washington, DC, U. S.) 2012, 112, 5641-5674.

(22) Griffin, G. B.; Young, R. M.; Ehrler, O. T.; Neumark, D. M. Electronic Relaxation Dynamics in Large Anionic Water Clusters: $\left(\mathrm{H}_{2} \mathrm{O}\right)_{n}^{-}$and $\left(\mathrm{D}_{2} \mathrm{O}\right)_{n}^{-}(\mathrm{n}=25-200)$. J. Chem. Phys. 2009, 131, 194302.

(23) Ma, L.; Majer, K.; Chirot, F.; von Issendorff, B. Low Temperature Photoelectron Spectra of Water Cluster Anions. J. Chem. Phys. 2009, 131, 144303.

(24) Coe, J. V.; Lee, G. H.; Eaton, J. G.; Arnold, S. T.; Sarkas, H. W.; Bowen, K. H.; Ludewigt, C.; Haberland, H.; Worsnop, D. R. Photoelectron Spectroscopy of Hydrated Electron Cluster Anions, $\left(\mathrm{H}_{2} \mathrm{O}\right)_{n=2-69}^{-}$. J. Chem. Phys. 1990, 92, 3980-3982.

(25) Young, R. M.; Yandell, M. A.; King, S. B.; Neumark, D. M. Thermal Effects on Energetics and Dynamics in Water Cluster Anions $\left(\mathrm{H}_{2} \mathrm{O}\right)_{n}^{-}$. J. Chem. Phys. 2012, 136, 094304.

(26) Barnett, R. N.; Landman, U.; Cleveland, C. L.; Jortner, J. Electron Localization in Water Clusters. II. Surface and Internal States. J. Chem. Phys. 1988, 88, 4429-4447.

(27) Madarász, Á.; Rossky, P. J.; Turi, L. Interior- and Surface-Bound Excess Electron States in Large Water Cluster Anions. J. Chem. Phys. 2009, 130, 124319.

(28) Verlet, J. R. R.; Bragg, A. E.; Kammrath, A.; Cheshnovsky, O.; Neumark, D. M. Comment on "Characterization of Excess Electrons in Water-Cluster Anions by Quantum Simulations". Science 2005, 310, $1769 \mathrm{~b}-1769 \mathrm{~b}$.

(29) Turi, L.; Sheu, W.-S.; Rossky, P. J. Response to Comment on "Characterization of Excess Electrons in Water-Cluster Anions by Quantum Simulations". Science 2005, 310, 1769c-1769c.

(30) Madarász, Á.; Rossky, P. J.; Turi, L. Response of Observables for Cold Anionic Water Clusters to Cluster Thermal History. J. Phys. Chem. A 2010, 114, 2331-2337.

(31) Kimura, Y.; Alfano, J. C.; Walhout, P. K.; Barbara, P. F. Ultrafast Transient Absorption Spectroscopy of the Solvated Electron in Water. J. Phys. Chem. 1994, 98, 3450-3458.

(32) Yokoyama, K.; Silva, C.; Son, D. H.; Walhout, P. K.; Barbara, P. F. Detailed Investigation of the Femtosecond Pump-Probe Spectroscopy of the Hydrated Electron. J. Phys. Chem. A 1998, 102, 69576966. 
(33) Assel, M.; Laenen, R.; Laubereau, A. Dynamics of Excited Solvated Electrons in Aqueous Solution Monitored with Femtosecond-Time and Polarization Resolution. J. Phys. Chem. A 1998, 102, 2256-2262.

(34) Pshenichnikov, M. S.; Baltuska, A.; Wiersma, D. A. HydratedElectron Population Dynamics. Chem. Phys. Lett. 2004, 389, 171-175.

(35) Thaller, A.; Laenen, R; Laubereau, A. Femtosecond Spectroscopy of the Hydrated Electron: Novel Features in the Infrared. Chem. Phys. Lett. 2004, 398, 459-465.

(36) Weber, J. M.; Kim, J.; Woronowicz, E. A.; Weddle, G. H.; Becker, I.; Cheshnovsky, O.; Johnson, M. A. Observation of Resonant Two-Photon Photodetachment of Water Cluster Anions via Femtosecond Photoelectron Spectroscopy. Chem. Phys. Lett. 2001, 339, 337-342.

(37) Elkins, M. H.; Williams, H. L.; Shreve, A. T.; Neumark, D. M. Relaxation Mechanism of the Hydrated Electron. Science 2013, 342, 1496-1499.

(38) Karashima, S.; Yamamoto, Y.; Suzuki, T. Resolving Nonadiabatic Dynamics of Hydrated Electrons Using Ultrafast Photoemission Anisotropy. Phys. Rev. Lett. 2016, 116, 137601.

(39) Borgis, D.; Rossky, P. J.; Turi, L. Quantized Time Correlation Function Approach to Nonadiabatic Decay Rates in Condensed Phase: Application to Solvated Electrons in Water and Methanol. J. Chem. Phys. 2006, 125, 064501.

(40) Borgis, D.; Rossky, P. J.; Turi, L. Nuclear Quantum Effects on the Nonadiabatic Decay Mechanism of an Excited Hydrated Electron. J. Chem. Phys. 2007, 127, 174508.

(41) Schwartz, B. J.; Rossky, P. J. Aqueous Solvation Dynamics with a Quantum Mechanical Solute: Computer Simulation Studies of the Photoexcited Hydrated Electron. J. Chem. Phys. 1994, 101, 69026916.

(42) Kee, T. W.; Son, D. H.; Kambhampati, P.; Barbara, P. F. A Unified Electron Transfer Model for the Different Precursors and Excited States of the Hydrated Electron. J. Phys. Chem. A 2001, 105, 8434-8439.

(43) Elkins, M. H.; Williams, H. L.; Neumark, D. M. Isotope Effect on Hydrated Electron Relaxation Dynamics Studied with TimeResolved Liquid Jet Photoelectron Spectroscopy. J. Chem. Phys. 2016, 144, 184503.

(44) Rizzuto, A. M.; Irgen-Gioro, S.; Eftekhari-Bafrooei, A.; Saykally, R. J. Broadband Deep UV Spectra of Interfacial Aqueous Iodide. J. Phys. Chem. Lett. 2016, 7, 3882-3885.

(45) Thürmer, S.; Seidel, R.; Faubel, M.; Eberhardt, W.; Hemminger, J. C.; Bradforth, S. E.; Winter, B. Photoelectron Angular Distributions from Liquid Water: Effects of Electron Scattering. Phys. Rev. Lett. 2013, 111, 173005.

(46) Signorell, R.; Goldmann, M.; Yoder, B. L.; Bodi, A.; Chasovskikh, E.; Lang, L.; Luckhaus, D. Nanofocusing, Shadowing, and Electron Mean Free Path in the Photoemission from Aerosol Droplets. Chem. Phys. Lett. 2016, 658, 1-6.

(47) Uhlig, F.; Herbert, J. M.; Coons, M. P.; Jungwirth, P. Optical Spectroscopy of the Bulk and Interfacial Hydrated Electron from $\mathrm{Ab}$ Initio Calculations. J. Phys. Chem. A 2014, 118, 7507-7515. 